\title{
Quasi-Interpolation by Means of Filter-Banks
}

\author{
Gerardo Pérez-Villalón
}

\begin{abstract}
We consider the problem of approximating a regular function $f(t)$ from its samples, $f(n T)$, taken in a uniform grid. Quasi-interpolation schemes approximate $f(t)$ with a dilated version of a linear combination of shifted versions of a kernel $\varphi(t)$, specifically $f_{a \mathrm{ppr} r x}^{T}(t)=\sum u_{f}[u] \varphi(t / T-u)$, in a way that the polynomials of degree at most $L-1$ are recovered exactly. These approximation schemes give order $L$, i.e., the error is $O\left(T^{L}\right)$ where $T$ is the sampling period. Recently, quasi-interpolation schemes using a discrete prefiltering of the samples $f(n T)$ to obtain the coefficients $a_{f}[\cdots]$, have been proposed. They provide tight approximation with a low computational cost. In this work, we generalize considering rational filter banks to prefilter the samples, instead of a simple filter. This generalization provides a greater flexibility in the design of the approximation scheme. The upsampling and downsampling ratio $r$ of the rational filter bank plays a significant role. When $r=1$, the scheme has similar characteristics to those related to a simple filter. Approximation schemes corresponding to smaller ratios give less approximation quality, bul, in return, they have less computational cost and involve less storage load in the system.
\end{abstract}

Index Terms-Approximation order, filter bank, quasi-interpolation, shift-invariant spaces, Strang-Fix conditions.

\section{INTRODUCTION}

$\mathbf{T}$ HIS work deals with the problem of approximating a regular function $f(t)$ from its samples taken in a uniform grid $\{f(n T)\}_{n \in \mathbb{Z}}$. In many approximation schemes, the function $f(t)$ is approximated by

$$
f_{\mathrm{approx}}^{T}(t)=\sum_{n \in \mathbb{Z}} a[n] \varphi\left(\frac{t}{T}-n\right)
$$

where $\varphi$ is a suitable kernel and the coefiicients $a[n]$ are obtained by a discrete filtering of the sequence of samples $\dot{s}_{j}[n]=$ $f(n T)$

$$
a[n]=\left(s_{f} * g\right)[n]
$$

with an advisable filter $g$. Equivalently

$$
f_{\text {approx }}^{T}(t)=\sum_{n \in \mathbb{Z}} f(n T) S\left(\frac{t}{T}-n\right)
$$

where $S(t)=\sum_{n \in \mathbb{Z}} g[n] \varphi(t-n)$ is the reconstruction function.

Manuscript recejved April 24, 2009; accepted October 21, 2009. First published November 20.2009; curtent version published Febiuary 10, 2010. The associate editor coordinating the review of this manuscript and approving it for publication was Prof. Haldun Ozaktas. This work was supported by the D.G.I. of the Spanish Ministerio de Ciencia e Innovación (MICINN) under Grant MTM2009-08345.

The author is with the Departamento de Matemática Aplicada a la Telecomunicación, E.U.I.T.T, Universidad Politécnica de Madrid, 28031 Madrid. Spain (e-Inail: gperez@euitt.upn.es).

Dişital Object Identifier 10.1109/TSP.2009.2037063
For interpolation schemes (see [1]-[5]), the interpolation condition

$$
f_{\text {approx }}^{T}(n T)=f(n T), \quad n \in \mathbb{Z}
$$

is required, and then the prefilter is necessarily given by $G(z)=$ $\left(\sum \varphi(n) z^{-n}\right)^{-1}$, where $G(z)$ denotes the z-transfonn of $g[n]$, $G(z)=\sum_{n \in \mathbb{Z}} g[n] z^{-n}$. The classical example is the Shannon sampling formula, where $\varphi(t)=S(t)=\operatorname{sinc}(t)$, which exactly recovers a bandlimited function $f(t)$ taking a small enough sampling period. This formula gives a tight approximation for functions that are approximately bandlimited. The slow decay of $\operatorname{sinc}(t)$ is its main inconvenience. A classical interpolating alternative is to consider a kernel $\varphi$ of compact support, satisfying the Strang-Fix conditions of order $L$, i.e.,

$$
\begin{aligned}
\hat{\varphi}(0) & \neq 0 \\
\hat{\varphi}^{(l)}(n) & =0, l=0,1, \ldots, L-1, n \in \mathbb{Z} \backslash\{0\}
\end{aligned}
$$

where $\hat{\varphi}(w)=\int \varphi(t) e^{-2 \pi i w t} d t$ denotes the Fourier transform. These conditions imply, under appropriate hypotheses, that the interpolation formula (3) exactly recovers any polynomial in $\Pi_{L-1}$, the space of polynomials of degree less than or equal to $L-1$. A suitable choice for the kernel $\varphi$, e.g., a B-spline (see [6]), allows us to efficiently compute the approximation by using (2) and (1) since $g[n]$ decays exponentially fast and $\varphi$ has compact support. Besides, the ability of reproducing polynomials in $\Pi_{L-1}$ guarantees that the interpolation scheme has approximation order $L$; specifically, for any $f$ in the Sobolev space $W_{2}^{L}(\mathbf{R}):=\left\{f:\left\|f^{(l)}\right\|_{2}<\infty, l \leq L\right\}$ (see [2], [7], and [8])

$$
\left\|f-f_{\text {approx }}^{T}\right\|_{2}=O\left(T^{L}\right)
$$

and for any $f$ in $W_{\infty}^{L}(\mathbf{R}):=\left\{f^{\prime}:\left\|f^{(l)}\right\|_{\infty}<\infty, l \leq L\right\}$ (see [8] and [9]),

$$
\left\|f-f_{\text {approx }}^{T}\right\|_{\infty}=O\left(T^{L}\right) .
$$

Quasi-interpolation schemes (see [7], [8], [10], and [11]) of order $L$ require only that the formula (3) reproduces polynomials in $\Pi_{L-1}$ (quasi-interpolation condition), which allows us to maintain the approximation order (6). This is a weaker condition than the interpolation condition (4), giving more flexibility for the choice of the filter $g$. For instance, if we look for a finite-impulse response (FIR) filter $G(z)$, the only condition that has to be satisfied is that the non-1ull coeficients of the filter $G(z)$ satisfy a certain linear system of $L$ equations (see [7] and [11]). Thus, in general, we can design FIR filters $g$ with a support of size $L$ satisfying the aforementioned condition. It is important to note the possibility of designing quasi-interpolation schemes, with an approximation quality close to that given by the orthogonal projector onto $V_{T}(\varphi)=\left\{\sum a[n] \varphi(t / T-n)\right.$ : $\left.a \in \ell^{2}\right\}$ (see [7] and [12]-[14]). 


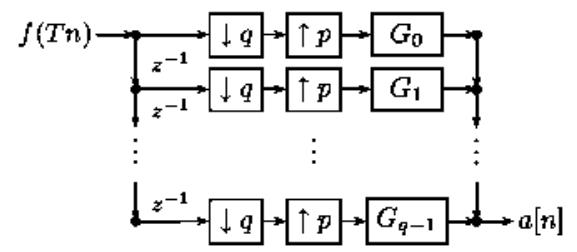

Fig. 1. Block diagram of the proposed prefiltering of the samples.

$$
f(T n) \rightarrow \uparrow p \rightarrow H \rightarrow \downarrow q \rightarrow u[n]
$$

Fig. 2. Prefiltering when $p$ and $q$ are coprime.

The interpolation and quasi-interpolation schemes have relcvant applications in image processing, such as rescaling, volume rendering, evaluating gradients, translations, rotations, and in gencral, geometric transformations of the signal (sce [4] and references (herein).

In this work, we study a generalization of the aforementioned quasi-interpolation schemes. We consider the possibility of obtaining quasi-interpolation schemes of order $L$ by using a rational filter bank instead of a simple filter. Specifically, we select two natural numbers, $q$ and $p$, and $q$ discrete filters, $g_{0}, g_{1}, \ldots, g_{q-1}$. The samples are filtered wilh a filter bank as shown in Fig. 1, yiclding to a cocfficient scquencc $a[n]$, and then the approximation is obtained by using

$$
\int_{\text {approx }}^{T}(l)=\sum_{n \in \mathbb{Z}} a[n] \varphi\left(\frac{p}{q T} l-n\right) .
$$

When $p$ and $q$ are coprime, the prefiltering can be easier represented by using the filter $H(z)=\sum_{j=0}^{q-1} z^{-j p} G_{j}\left(z^{q}\right)$ as shown in Fig. 2. Reciprocally, any prefiltering of the lype given in Fig. 2 can be represented by using the filters $g_{j}[n]=h[n q+j p], j=$ $0, \ldots, q-1$, as shown in Fig. 1 (see Section II). The representation of the prefiltering with $H(z)$ is simpler than the representation with the filters $G_{j}(z)$. However, the reconstruction functions, the quasi-interpolation condition, and the error for this approximation scheme, except in the case $p=1$, admit a simpler expression in terms of the filters $G_{j}(z)$.

The proposed prefiltering implies a rational rate change, where the upsampling and downsampling ratio is $r=p / q$. Thus, we have the possibility of choosing the ratio $r$ according to the desirable characteristics of the approximation scheme. If $r=1$ the scheme has similar characteristics to those related to a simple filter, in fact when $p=q=1$, it is one of them. If $r<1$, the approximation scheme, as a consequence of the compression, loses in quality approximation (see Section IV), but it gains in numerical efficiency since the computational cost of the algorithm is approximately proportional to $\tau$ (see Sections II and III). Note that the compression associated with a ratio $r<1$ is also suitable in order to transmit or store the signal. From the practical point of view, the case of $\tau>1$, although theoretically possible, is not interesting.

In [15], one can find conditions on the filters $G_{j}$ (with a slight change of notation) that guarantee the perfect reconstruction of the functions belonging to $V_{T}(\varphi)$. This perfect reconstruction is not required here; it is only required for polynomials in $\Pi_{L-1}$ (a weaker condition). Thus, we gain in flexibility, allowing us to design approximation schemes involving fillers with a smaller support.

In [16], a variational method that applies to this setting is given. It allows to approximate a function from nonuniform samples. For $r<1$ and without variational penalty, the method gives the function in $V_{T}(\varphi)$ that is the elosest to the samples in the $\ell^{2}$ sense.

For the sake of simplicity, in this work, we confine ourselves to FIR filters. However, most of the results are valid to IIR filters (with suitable decay conditions). For the case of $r=1$, in [7], [12], and [14] are given IIR filter satisfying the quasi-interpolating condition, which can be implemented by a fast algorithm. See [13] for the case of $r=1 / q$.

The paper is organized as follows. In Section II, we introduce the proposed approximation scheme. In Section III, we deduce a necessary and sufficient condition [see infra (16)] on this scheme to reproduce polynomials of degrec at most $L-1$ (quasi-interpolation condition), and we show that by solving a linear system of equations, it is possible to obtain FIR filters $G_{j}$ salisfying this condition. In Section IV, we give examples of such FIR filters. In Section V, we prove that whenever the aforementioned condition holds, the scheme has approximation order $L$ in both the $L^{2}(\mathbb{R})$ and $L^{\infty}(\mathbb{R})$-norm sense. In this section, by applying the стог formula given in [10], we get an expression for the approximation error, which gives us a suitable criterion in order to choose among the filters $G_{j}$ that satisfy the quasi-interpolation condition. In Section VI, we give simulations showing the behavior of the approximation technique for different values of the ratio $r$. We conclude in Section VII. An Appendix includes the proofs of the results given in Sections I, II, and V.

\section{The Approximation Scheme}

Throughout this paper, we assume that $\varphi$ is a continuous real function, compactly supported, that satisfies the Strang-Fix conditions of order $L(5)$. We also assume that the sequence $\{\varphi(t-n)\}_{n \in \mathbb{Z}}$ is a Riesz basis for the space $V_{1}(\varphi)=$ $\left\{\sum a[n] \varphi(t-n): a \in \ell^{2}\right\}$; equivalently, there exist constants $A, B$ such that $0<A \leq \sum_{n \in \mathbb{Z}}|\hat{\varphi}(w+n)|^{2} \leq B<\infty$. The dual function $\varphi_{d}$ is defined by

$$
\hat{\varphi}_{d}(w):=\frac{\hat{\varphi}(w)}{\sum_{n \in \mathbb{Z}}|\hat{\varphi}(w+n)|^{2}} .
$$

Discrete filters are either described by their impulse response $g[n]$ or by their $z$-transform $G(z)=\sum_{n \in \mathbb{Z}} g[n] z^{-n}$ for which we use uppercase symbols.

Associated with the kernel $\varphi$, two natural numbers $p$ and $q$, and $q$ FIR filters $g_{0}, g_{1}, \ldots, g_{g-1}$, we consider the approximation scheme

$$
\begin{aligned}
a[n] & =\sum_{j=0}^{q-1} \sum_{k \in \mathbb{Z}} f(q k T-j T) g_{j}[n-k p] \\
f_{\text {approx }}^{T}(t) & =Q_{T} f(t):=\sum_{n \in \mathbb{Z}} a[n] \varphi\left(\frac{r}{T} t-n\right) .
\end{aligned}
$$

where $r$ denotes the ratio $r:=p / q$.

Thus, to approximate a function $f(t)$ from its samples $f(n T)$, we first prefilter the samples with the filter bank shown in Fig. 1, 
which yields to a coefficient sequence $a[n]$, and then we apply $f_{\text {approx }}^{T}(t)=\sum a[n] \varphi(r t / T-n)$.

Another possibility, yielding to the same approximation $f_{\text {approx }}^{T}$, is to evaluate

$$
f_{\text {approx }}^{T}(t)=\sum_{j=0}^{q-1} \sum_{n \in \mathbb{Z}} f(q n T-j T) S_{j}\left(\frac{r}{T} t-n p\right)
$$

where the reconstruction functions are defined by

$$
S_{j}(t):=\sum_{n \in \mathbb{Z}} g_{j}[n] \varphi(t-n), \quad j=0,1, \ldots, q-1 .
$$

When $p$ and $q$ are coprime, the prefiltering ( 9 ) can be also represented as (see Fig. 2)

$$
a[n]=\sum_{k \in \mathbb{Z}} f(k T) h(q n-p k)
$$

where $H(z)$ is defined by

$$
H(z):=\sum_{j=0}^{q-1} z^{-j p} G_{j}\left(z^{q}\right)
$$

The proof is given in the Appendix. There, it is also proved that (13) is equivalent to

$$
g_{j}[n]=h[n q+j p], \quad n \in \mathbb{Z}, j=0, \ldots, q-1 .
$$

Hence, for any FIR filter $h . n]$, the prefiltering (12) can be represented as (9), where the filters $g_{j}[n]$ are defined by (14).

See references [17] and [18] for efficient structures for fractional decimation and for filter banks.

The (type 2) polyphase components $R_{j, k}$ of the filters $G_{j}$ are defined by

$$
G_{j}(z)=\sum_{k=0}^{p-1} z^{-(p-1-k)} R_{k, j}\left(z^{p}\right), \quad j=0,1, \ldots, q-1 .
$$

Note that, under the assumed hypotheses, $f_{\text {approx }}^{T}(t)$ is well defined for any function $f$. Besides, the reconstruction functions $S_{j}$ have compact support.

The ratio $r$ plays and important role in the numerical efficiency of the reconstruction algorithm (9) and (10). Indeed, the computational cost of the second step of the algorithm (to evaluate $\left.\sum a[n] \varphi(r t / T-n)\right)$ is proportional to the number of coefficients $a[n]$ considered, which is approximately equal to $r \times N$, where $N$ is the number of available samples (assuming that $N$ is much bigger than the size of the support of the filters $g_{j}$ ). In the next section, we show that if we choose the filters with the smallest support, the computational cost of the first step (the prefiltering) of the algorithm is also proportional to $r$. The cost of this first step is almost negligible compared to the second one (see [1] and [5]), which requires evaluations of the kernel.

Note that except in the case of $q=1$, the system does not satisfy the shift invariant property $Q_{T}[f(\cdot-T n)](t)=Q_{T} f(t-$ $T n)$, but it satisfies $Q_{T}[f(\cdot-T q n)](t)=Q_{T} f(t-T q n)$.

\section{ThE QUAASI-INTERPOLATION CONDITION}

We denote $\mathbf{G}(z):=\left[G_{0}(z), G_{1}(z), \ldots G_{q-1}(z)\right]^{\top}$. As usual, $\xi(w)=O\left(w^{L}\right)$ means that $\left|\xi(w) / w^{L}\right|$ is bounded as $w \rightarrow 0$ (when $\xi \in C^{\infty}(\mathbb{R})$, it is equivalent to $\xi^{(t)}(0)=0$ for $l=0,1, \ldots, L-1) ; \delta_{n}$ denotes the delta sequence; and $\bar{c}$ denotes the conjugate of $c \in \mathbb{C}$. Finally, let $\mathbf{e}_{l}(z)$, for $l=p, q$, denote

$$
\mathrm{e}_{l}(z)=\left[1, z^{-1}, \ldots z^{-(l-1)}\right]^{\top}
$$

Recall that we have assumed that the kernel $\varphi(t)$ satisfies the Strang-Fix conditions of order $L(5)$.

The next lemma gives a necessary and sufficient condition on the approximation scheme (9) and (10) in order to reproduce polynomials of degree at most $L-1$.

Lemma 1: The approximation operator $Q_{T}$ defined in (9) and (10) satisfies $Q_{T} \rho=\rho$ for all $\rho \in \Pi_{L-1}$ if and only if the condition

$$
\mathbf{e}_{\eta}^{\top}\left(e^{2 \pi i r w}\right) \mathbf{G}\left(e^{2 \pi i(w+n / p)}\right) \hat{\varphi}\left(w+\frac{n}{p}\right)-p \delta_{n}=O\left(w^{L}\right)
$$

is satisfied for all $n \in \mathbb{Z}$.

The proofs of this and the next lemma are given in the Appendix. Next, we give a more manageable sufficient condition. Notice that since $\varphi$ has compact support, the function $A_{\varphi}(w)=\sum|\varphi(w+n)|^{2}$ is a trigonometric polynomial (see [19]), and then the function $\hat{\varphi}_{d}$ defined in (8) is infinitely differentiable.

Lemma 2: Any of the equivalent conditions

$$
\mathbf{e}_{q}^{\top}\left(e^{2 \pi i r w}\right) \mathbf{G}\left(e^{2 \pi i(w+k / p)}\right)-p \overline{\hat{\varphi}}_{d}(w) \delta_{k}=O\left(w^{L}\right)
$$

for $k=0,1, \ldots, p-1$, or

$$
\left[\frac{d^{l}}{d w^{l}} \sum_{j=0}^{q-1} R_{k, j}\left(e^{2 \pi i p w}\right) e^{-2 \pi i(j r+p-1-k) w}\right]_{w=0}=\bar{\varphi}_{d}^{(l)}(0)
$$

for $k=0,1, \ldots, p-1$, and for $l=0,1, \ldots, L-1$, where $R_{k, j}(z)$ are the polyphase components defined in (15), implies that condition (16) holds or equivalently that $Q_{T} \rho=\rho$ for all $\rho \in \Pi_{L-1}$.

For the case of $r^{*}=p=q=1$, condition (17) reduces to

$$
\mathbf{G}\left(e^{2 \pi i w}\right)=\overline{\hat{\varphi}}_{d}(w)+O\left(w^{L}\right)
$$

and it can be found in [7], [11], and [12]. Thus, in order to find a FIR filter $g=g_{0}$ so that the approximation scheme (3) reproduces the polynomials in $\Pi_{L-1}$, we have to solve a linear system with $L$ equations whose unknowns are the non-null $g[n]$. Thus, in general, it is possible to find a FIR filter $g$ with support of size $L$ satisfying the reproducing condition. If we consider $N$ samples, this filter implies approximately $L \times N$ sums and products in the prefiltering step (assuming that $N$ is much bigger that $L$ ).

In the general case, in order to find $q$ FIR filters $g_{0}, g_{1}, \ldots, g_{4-1}$, such that the approximation scheme (9) and (10) reproduces the polynomials of $\Pi_{L-1}$, we have to solve a linear system with $p \times L$ [see (17) or (18)] whose unknowns are the non-null $g_{j}[n]$. Thus, it is possible, in general, to find FIR filters $g_{j}$ being the sum of the size of their supports equal to $p \times L$ and satisfying the reproducing condition. For these filters, the computation of the prefiltering step (9) involves approximately $r \times L \times N$ products and sums; hence, the resulting computational cost of the prefiltering step is approximately proportional to $r$. 
If the kernel $\varphi$ is even (recall that we have supposed that it is real), a suitable property (see [4]), then $\hat{\varphi}_{d}^{(l)}(t)$ is real and even, and thus $\hat{\varphi}_{d}^{(l)}(0)$ is real and is equal to 0 when $l$ is odd. As a consequence, condition (18) has real solutions, which implies real filters.

In the case of $r=1 / q$, condition (17) reads as

$$
\left[\frac{d^{l}}{d u^{l}} H\left(e^{2 \pi i w / q}\right)\right]_{u=0}=\overline{\hat{\varphi}}_{d l}^{(l)}(0), \quad l=0, \ldots, L-1
$$

where $H(z)$ is given by (13). Note that if the kernel $\varphi$ is even, then any even filter $h(n)$ satisfies the above equations for $l$ odd. Thus, we can achieve symmetric approximation schemes, with a filter of support $L$ when $L$ is odd, and of support $L-1$ when $L$ is even.

As the reader can observe, in the proof of Lemma 2, conditions (17) and (18) are almost always necessary for the reproduction property: $Q_{T} \rho=\rho$ for all $\rho \in \Pi_{L-1}$.

\section{Examples With the Quadratic B-SPline}

In order to illustrate the theoretical results, we consider as an example the generator

$$
\varphi(t)=\beta^{2}(t)= \begin{cases}\frac{3}{4}-t^{2}, & -\frac{1}{2} \leq t<\frac{1}{2} \\ \frac{1}{2}\left(|t|-\frac{3}{2}\right)^{2}, & \frac{1}{2} \leq|t|<\frac{3}{2} \\ 0, & |t| \geq \frac{3}{2}\end{cases}
$$

the centered quadratic B-spline, which satisfies the Strang-Fix conditions of order $L=3$. We have (see [7])

$$
\hat{\varphi}_{d}(w)=\frac{\sin ^{3}(\pi w)}{\pi^{3} w^{3}\left[1-\sin ^{2}(\pi w)+\left(\frac{2}{15}\right) \sin ^{4}(\pi w)\right]} .
$$

In the case of $r=1$, the unique filter of the type $H(z)=$ $a+b\left(z+z^{-1}\right)$ that satisfies (19) is

$$
H_{1}(z):=\frac{5}{4}-\frac{1}{8}\left(z+z^{-1}\right) .
$$

The approximation algorithm, (12) and (10), reads

$$
\begin{aligned}
a[n] & =\frac{5}{4} f(n T)-\frac{1}{8}[f(n T+T)+f(n T-T)] \\
f_{\text {approx }}^{T}(t) & =\sum_{n \in \mathbb{Z}} a[n] \beta^{2}\left(\frac{t}{T}-n\right) .
\end{aligned}
$$

In the case of $r=1 / 2$, the filter

$$
H_{1 / 2}(z):=2-\frac{1}{2}\left(z+z^{-1}\right)
$$

is a solution of (19). The approximation algorithm now reads

$$
\begin{aligned}
a[n] & =2 f(2 n T)-\frac{1}{2}[f(2 n T-T)+f(2 n T+T)] \\
f_{\text {approx }}^{T}(t) & =\sum_{n \in \mathbb{Z}} a[n] \beta^{2}\left(\frac{t}{2 T}-n\right) .
\end{aligned}
$$

In the case of $r=2 / 3$, the vector of filter

$$
\mathbf{G}_{2 / 3}(z):=\left[\begin{array}{c}
\frac{25}{16}-\frac{13}{64}\left(z+z^{-1}\right) \\
\frac{45}{64} z^{-1}-\frac{9}{32} \\
-\frac{9}{32} z^{-2}+\frac{43}{64} z^{-1}
\end{array}\right]
$$

satisfies condition (17). The algorithm (9)-(10) reads

$$
\begin{aligned}
a[2 n]= & \frac{25}{16} f(3 n T)-\frac{9}{32}[f(3 n T+T)+f(3 n T-T)] \\
a[2 n+1]= & -\frac{13}{64}(f(3 n T)+f(3 T n+3 T)) \\
& +\frac{45}{64}[f(3 n T+T)+f(3 n T+2 T)] \\
f_{\text {approx }}^{T}(t)=\sum_{n \in \mathbb{Z}} & {\left[a[2 n] \beta^{2}\left(\frac{2 t}{3 T}-2 n\right)\right.} \\
& \left.+a[2 n+1] \beta^{2}\left(\frac{2 t}{3 T}-2 n-1\right)\right] .
\end{aligned}
$$

In order to fix the suppor of the entries of $\mathbf{G}_{2 / 3}$, we have taken into account that $a[2 n+1]$ is the coefficient of $\beta^{2}((2 /(3 T))[t-$ $3 n T-3 T / 2]$ ), which is centered at $3 n T+3 T / 2$, and $a[2 n]$ is the coefficient of $\beta^{2}((2 /(3 T))[t-3 n T])$, which is centered at $3 n T$.

In the case of $r=3 / 4$, the vector of filters

$$
\mathbf{G}_{3 / 4}(z):=\left[\begin{array}{c}
\frac{13}{9}-\frac{17}{81}\left(z+z^{-1}\right) \\
-\frac{2}{9}+\frac{26}{2 \bar{z}} z-\frac{10}{81} z^{2} \\
\frac{10}{27}\left(z+z^{2}\right) \\
-\frac{10}{81} z+\frac{26}{25} z^{2}-\frac{2}{9} z^{3}
\end{array}\right]
$$

satisfies (17). Its corresponding prefiltering step is

$$
\begin{aligned}
a[3 n]= & \frac{13}{9} f(4 T n)-\frac{2}{9}(f(4 T n-T)+f(4 n T+T)) \\
a[3 n+1]= & -\frac{17}{81} f(4 T n)-\frac{10}{81} f(4 T n+3 T) \\
& +\frac{10}{27} f(4 T n+2 T)+\frac{26}{27} f(4 T n+T) \\
a[3 n+2]= & -\frac{17}{81} f(4 T n+4 T)+\frac{26}{27} f(4 T n+3 T) \\
& +\frac{10}{27} f(4 T n+2 T)-\frac{10}{81} f(4 T n+T) .
\end{aligned}
$$

Let us recall that the interpolating filter (such that (4) is satisfied) for the generator $\varphi=\beta^{2}$ is

$$
H^{\mathrm{int}}(z):=\frac{1}{\frac{3}{4}+\frac{1}{8}\left(z+z^{-1}\right)} .
$$

Finally, let us give an interesting example from [13] of an IIR filter satisfying the quasi-interpolating condition that can be implemented with a fast algorithm. This example is for the case of $\varphi=\beta^{2}$ and $r=1 / 2$. It is

$$
H^{\mathrm{IIR}}(z):=\frac{z^{-1}+z+2}{\frac{5}{2}+\frac{3}{4}\left(z^{2}+z^{-2}\right)} .
$$

Examples for other B-splines $\beta^{n}$ and other values of $r=1 / q$ can be found in [13].

\section{THE APPROXIMATON ERROR}

We split this section into three subsections. In the first one, we obtain a pointwise estimation for the approximation error and study the $L^{\infty}$-norm of this error. In the second one, we apply the results in [10] to study the $L^{2}$-norm of the error. The third 
one is concerned with designing the filters $G_{j}$ in order to get a smaller $L^{2}$-approximation crror.

\section{A. A Pointwise Estimation and the $L^{\infty}$-Approximation Error}

Theorem 1 proves that whenever the quasi-interpolation condition (16) holds, the approximation scheme has approximation order $L$ in the $L^{\infty}$-norm sense. Besides, it gives a pointwise estimation for the error, which shows that the error depends on the compression factor $r$. We denote

$$
\begin{aligned}
& \Omega(t):=(-1)^{L} \sum_{j=0}^{q-1} \sum_{n \in \mathbb{Z}}(t-p n+j r)^{L} S_{j}(t-p n) \\
& \widetilde{\Omega}(t):=\sum_{j=0}^{q-1} \sum_{n \in \mathbb{Z}}\left|(t-p n+j r)^{L} S_{j}(t-p n)\right| .
\end{aligned}
$$

Note that since the reconstruction functions $S_{j}$ are continuous and compactly supported, $\Omega$ and $\widetilde{\Omega}$ are bounded functions.

Theorem 1: Whenever the condition (16) holds, for all $f \in$ $W_{\infty}^{L}(\mathbb{R})$, we have

$$
\left\|f_{\text {approx }}^{T}-f\right\|_{\infty} \leq \frac{\|\widetilde{\Omega}\|_{\infty}\left\|f^{(L)}\right\|_{\infty}}{L !} \frac{T^{L}}{r^{L}}
$$

and, for all $f \in W_{\infty}^{L+1}(\mathbf{R})$, we have

$$
f_{\text {approx }}^{T}(t)-f(t)=\frac{\Omega\left(\frac{r t}{T}\right) f^{(L)}(t)}{L !} \frac{T^{L}}{r^{L}}+O\left(T^{L+1}\right) .
$$

The proof, based on the Taylor polynomial of $f$, is given in the Appendix. For the case of $r=p=q=1$, the estimations (28) and (29) are given in [8].

\section{B. The $L^{2}$-Approximation Error}

In [10], Blu and Unser gave a formula for the $L^{2}$-norm of the approximation crror valid to an ample set of approximation operators $\mathcal{L}_{h}$; For any $f \in W_{2}^{k}(\mathbf{R})$

$$
\left\|f-\mathcal{L}_{h} f\right\|_{2}=\left[\int_{-\infty}^{\infty}|\hat{f}(w)|^{2} E(h w) d w\right]^{1 / 2}+O\left(h^{k}\right)
$$

where the integral kemel $E(w)$ depends on the approximation scheme but does not depend on $f$. Morcover, $\left[\int|\hat{f}(w)|^{2} E(h w) d w\right]^{1 / 2}$ gives exactly the average approximation error (see [10, Theorem 2]). This error formula (30) can also be applied to the operator $Q_{T}$ by changing the sampling step; specifically, it can be applied to $\mathcal{L}_{h}:=Q_{h r}$. In this case, the formula (30) can be expressed as

$\left\|f-Q_{T} f\right\|_{2}=\left[\frac{1}{T} \int_{-\infty}^{\infty}\left|\hat{f}\left(\frac{w}{T}\right)\right|^{2} E\left(\frac{w}{r}\right) d w\right]^{1 / 2}+O\left(h^{k}\right)$

where the integral kernel is (assuming that the filters $g_{j}$ are real)

$$
\begin{aligned}
E(w)=1 & -\frac{2}{p} \Re\left[\mathbf{e}_{p}^{\top}\left(z^{r}\right) \mathbf{G}(z) \hat{\varphi}(w)\right] \\
& +\frac{1}{p^{2}} \sum_{k=0}^{p-1}\left|\mathbf{e}_{\varphi}^{\top}\left(z^{r}\right) \mathbf{G}\left(z e^{2 \pi i k / p}\right)\right|^{2} A_{\varphi}\left(w+\frac{k}{p}\right)
\end{aligned}
$$

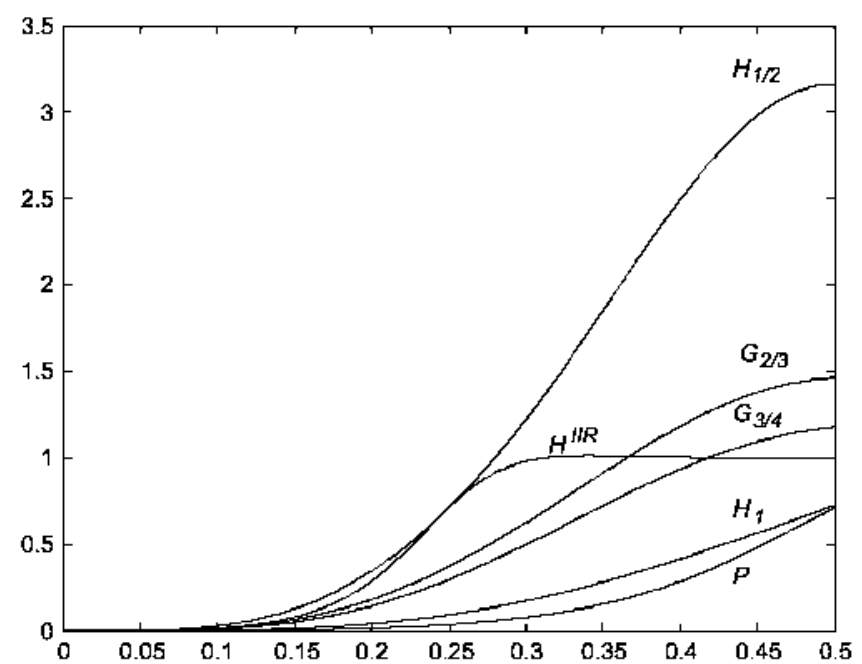

Fig. 3. $\sqrt{E(w / r)}$ for $H_{1}, G_{3 / 4}, G_{2 / 3}, H_{1 / 2}, H^{\mathrm{III}}$, and for the orthogonal projector $P(r=1$ in this case).

where $z=e^{2 \pi i w}$, $\Re$ denotes the real part, and $A_{\varphi}(w):=$ $\sum_{n \in \mathbb{Z}}|\hat{\varphi}(w+n)|^{2}$ (see the details in the Appendix). Fig. 3 shows the square root of the integral kernel $\sqrt{E(w / r)}$, corresponding to the orthogonal projector $P$ [see infra (35)] and to the filters $H_{1}, G_{3 / 4}, G_{2 / 3}, H_{1 / 2}$, and $H^{\mathrm{IIR}}$ defined in (20), (22), (24), (25), and (27), respectively. Fig. 4 shows $\sqrt{E(w / r)}$ for $P$, $H_{1}$, and for the interpolating filter $H^{\text {int }}$ defined in (26).

In [10], some results about the approximation error of the operator $\mathcal{L}_{h}$ are deduced from the formula (30). In Theorem 2, we collect the applications of these results to the operator $Q_{T}=$ $\mathcal{L}_{T / r}$. In particular, we deduce that the quasi-interpolation condition (16) in Lemma 1 is necessary and sufficient to the approximation scheme (9) and (10) to have order $L$ in the $L^{2}$-norm sense.

Note that since we have assumed that $\varphi$ has compact support (thus $A_{\varphi}(w)$ is a trigonometric polynomial) and $G_{j}(z)$ are FIR filters, the integral kernel $E(w)$ given in (32), has infinite bounded derivatives.

Theorem 2: Assume that $g_{j}$ are real FIR filters. The quasiinterpolating condition (16) holds if and only if

$$
\left\|f_{\text {approx }}^{T}-f\right\|_{2}=O\left(T^{L}\right), \quad \text { for all } f \in W_{2}^{L}(\mathbf{R}) .
$$

Morcover, in this casc, for all $f \in W_{2}^{L+1}(\mathbb{R})$

$\left\|f_{\text {ipprox }}^{T}-f\right\|_{2}=\frac{T^{L}}{r^{L}} \frac{\left\|f^{(L)}\right\|_{2}}{p(2 \pi)^{L} L !} \sqrt{\sum_{n \in \mathbb{Z}}\left|\Gamma_{n}^{(L)}(0)\right|^{2}}+O\left(T^{L+1}\right)$

where $\Gamma_{n}(w)=\mathbf{e}_{q}^{\top}\left(e^{2 \pi i r w}\right) \mathbf{G}\left(e^{2 \pi i(w+n / p)}\right) \hat{\varphi}(w+n / p)$, and for all $f \in W_{2}^{k}(\mathbb{R})$, with $k \geq L$

$$
\left\|f_{\text {ilpprox }}^{T}-f\right\|_{2} \leq C \frac{T^{L}}{r^{L}}\left\|f^{(L)}\right\|_{2}+K \frac{T^{k}}{r^{k}}\left\|f^{(k)}\right\|_{2}
$$

where

$$
C=\frac{\sqrt{\left\|E^{(2 L)}\right\|_{\infty}}}{2^{L} \pi^{L} \sqrt{(2 L) !}} \text { and } K=\frac{2 p^{k}}{\pi^{k}}\left[\|E\|_{\infty} \sum_{n \geq 1} n^{-2 k}\right]^{1 / 2} \text {. }
$$




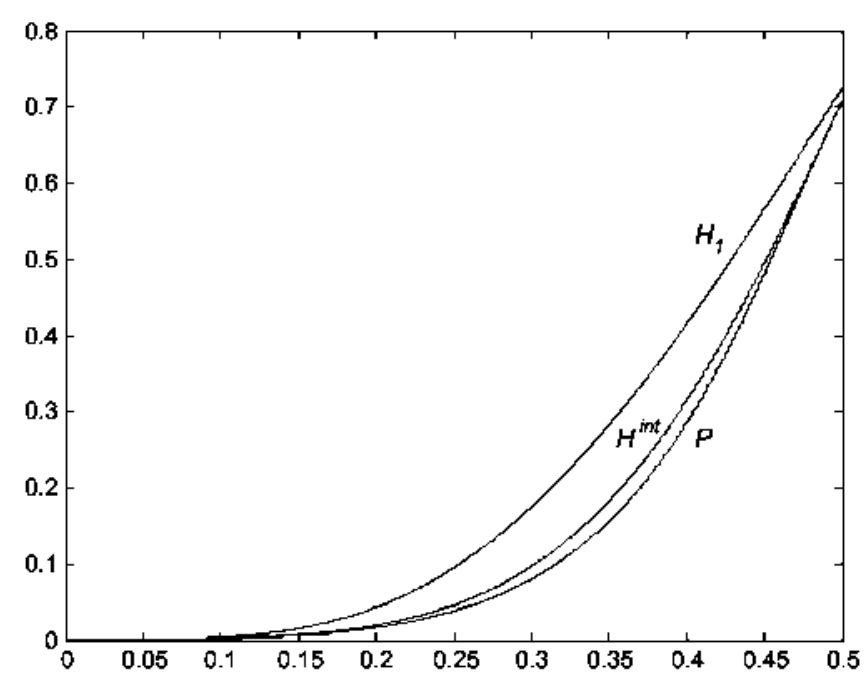

Hig. 4. $\sqrt{E(n / r)}$ for the onhogonal projector $P$, for the interpolating filter $H^{\text {int }}$, and for $H_{1}$.

To prove these results, we only have to apply the theorems in [10] (see the Appendix). When $r=1 / q$, the above expression for $\Gamma_{n}$ reduces to $\Gamma_{n}(w)=H\left(e^{2 \pi i w / q}\right) \hat{\varphi}(w+n)$. In the case of $r=p=q=1$, it reduces to $\Gamma_{n}(w)=\hat{S}(w+n)$. For this case, the estimation (33) is given in [8].

\section{Optimal Filters}

The orthogonal projector onto $V_{h}(\varphi)$ defined by

$$
P_{h} f(t):=\frac{1}{h} \sum_{n \in \mathbb{Z}} \int_{-\infty}^{\infty} f(\tau) \varphi_{d}\left(\frac{\tau}{h}-n\right) d \tau \varphi\left(\frac{t}{h}-n\right)
$$

gives the best approximation, in the $L^{2}$-norm sense, by a function in $V_{h}(\varphi)$. This approximation cannot be computed from the samples of the function. However, we can take advantage of the flexibility provided by the quasi-interpolation schemes to get an approximation whose error is close to that given by the orthogonal projector. To do this, we will base, as it is done in [7] and [12]-[14], on the error formula (30). The orthogonal projector $P_{h}$ satisfies the estimation (30) and the integral kemel, which we denote by $E_{\min }$ (see [7]), is

$$
E_{\min }(w)=1-\frac{|\hat{\varphi}(w)|^{2}}{A_{\varphi}(w)} .
$$

The integral kernel (32) can be expressed as

$$
E(w)=E_{\min }(w)+E_{\text {res }}(w)
$$

where

$$
\begin{aligned}
E_{\mathrm{res}}(w)= & A_{\varphi}(w)\left|\overline{\hat{\varphi}}_{d}(w)-\frac{1}{p} \mathbf{e}_{q}^{\top}\left(z^{r}\right) \mathbf{G}(z)\right|^{2} \\
& +\frac{1}{p^{2}} \sum_{k=1}^{p-1}\left|\mathbf{e}_{q}^{\top}\left(z^{r}\right) \mathbf{G}\left(z e^{2 \pi i k / p}\right)\right|^{2} A_{\varphi}\left(w+\frac{k}{p}\right)
\end{aligned}
$$

and $z=e^{2 \pi i w}$. The quasi-interpolation condition (17) gives

$\mathbf{e}_{q}^{\top}\left(z^{r}\right) \mathbf{G}\left(z e^{2 \pi i k / p}\right)-p \overline{\hat{\varphi}}_{d}(w) \delta_{k}=O\left(w^{K}\right), \quad k=0, \ldots, p-1$

holds for $K=L$, and then $E_{\mathrm{res}}(w)=O\left(|w|^{2 L}\right)$. Thus, whenever most of the energy of $\hat{f}(u / T)$ is concentrated close to zero, the approximation error is small. In order to do it smaller, we can try to find a vector of filters satisfying (38) for a bigger $K$. Besides, having in mind that a part of the energy of $\hat{f}(w / T)$ could not be so close to zero, we can also use the flexibility given for a bigger support of the filters in order to minimize [see (31) and (36)]

$$
\int_{-\infty}^{\infty} v(w) E_{\mathrm{res}}\left(\frac{w}{r}\right) d w
$$

where $v(w) \geq 0$ is a suitable weight. Namely, we fix a weight $v(w)$, an order $K \geq L$, and the support of the filters. Then, we search the $G$ that minimize (39) among those that satisfy (38). This is a linear problem easy to solve. For example, when $\varphi=\beta^{2}, r=1$, the symmetrical filters $I I(z)$ of the type

$$
H(z)=a+\frac{b}{2}\left(z+z^{-1}\right)+\frac{c}{2}\left(z^{2}+z^{-2}\right)
$$

satisfying (38) for $K=L+1=4$ are those given by

$$
a=\frac{5}{4}+3 c, \quad b=-\frac{1}{4}-4 c .
$$

Thus, minimizing (39) is equivalent to finding $c$ such that

$$
\begin{aligned}
\int_{\infty}^{\infty} v(w) \Lambda_{\varphi}(w) & \left(\hat{\varphi}_{d}(w)-\frac{5}{4}+\frac{1}{4} \cos (2 \pi w)\right. \\
& -c[3-4 \cos (2 \pi w)+\cos (4 \pi w)]) d w
\end{aligned}
$$

is minimum. For instance, when $v(w)$ is the characteristic function of the interval $(-1 / 4,1 / 4)$, then $c \approx 0.054$ and the searched filter is

$$
H_{1}^{o}(z):=1.412-0.233\left(z+z^{-1}\right)+0.027\left(z^{2}+z^{-2}\right) .
$$

For the rate $r=1 / 2$, the same criterium provides

$$
H_{1 / 2}^{o}(z):=2.87-1.08\left(z+z^{-1}\right)+0.145\left(z^{2}+z^{-2}\right) .
$$

Fig. 5 shows the square root of $E_{\text {res }}(w / r)$ for these filters as well as for those with minimum support. Note that the bigger support increases the computations in the prefiltering step but has no influence on the number of evaluations of the kernel.

\section{SimULATIONS}

In this section, we give numerical simulations showing the behavior of the approximation scheme given in Sections IV and V. Specifically, we apply the algerithms to recover the functions shown in Fig. 6 from 31 samples taken in the interval $[-3,3]$ 

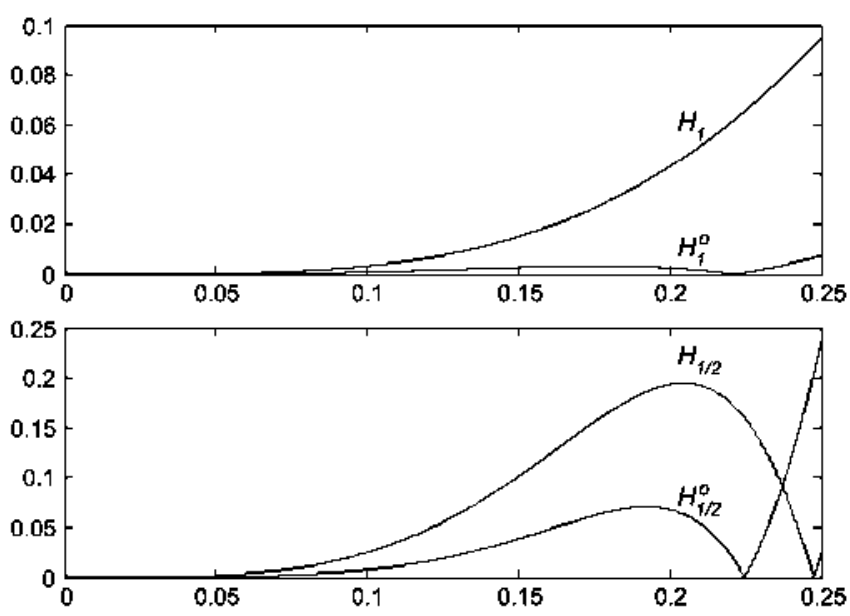

Fig. 5. $\sqrt{E_{\text {ros }}(w / r)}$ for $H_{1}, H_{1}^{\circ}, H_{1 / 2}$, and $H_{1 / 2}^{\circ}$

with period $T=0.2$. The $L^{2}[-3,3]$-norm of the error for the different algorithms and examples are given in Table $\mathrm{I}$.

For instance, for the first example $f(t)=(1-t) e^{-t^{2}}$, the algorithm (21) that does not entail compression $H_{1}$ (corresponding to $r=1$ ) gives an error of $7 \times 10^{-4}$. It needs 30 evaluations of the kernel. The algorithm conesponding to $H_{1 / 2}$ needs only 15 evaluations of the kernel, but its error increases to $6.4 \times 10^{-3}$. Between these cases are those corresponding to the rates $r=3 / 4$ and $r=2 / 3$.

For the second example, the errors are bigger (it has wide variations). The behavior with respect to the different rates are the same.

For the third example, the $L^{2}[-3,3]$-norm of the error, due fundamentally to the discontinuity, is approximately equal for all the rates. However, the reproduction of the discontinuity is better using a bigger $r$, as it is shown in Fig. 7 .

Table I also gives the errors for the interpolating filter $I^{\text {int }}$ and for the IIR filter $H^{\text {IIR }}$, which are close to those given by $H_{1}$ and $H_{1 / 2}^{o}$, respectively.

The pointwise error for the second example and for some of the algorithms can be seen in Fig. 8.

As can be observed in Table I, using the optimized filters $H_{1}^{o}$ and $H_{1 / 2}^{o}$ improves the results with respect to the minimum support fillers $H_{1}$ and $H_{1 / 2}$. The reconsiruction of the second example using $I_{1}$ and $I_{1}^{o}$ and sampling period $T=0 . \overline{5}$ can be seen in Fig. 9.

The gradient, used in some applications (c.g., contour detection, shading), can be computed by means of the coefficients $a[n]$ obtained in the prefiltering step (9). Indeed, using (10) and that $d / d t \beta^{2}(t)=\beta^{1}(x+1 / 2)-\beta^{1}(x-1 / 2)$, where $\beta^{1}$ is the B-spline of degree 1 , we obtain that

$$
\frac{d}{d t} f_{\text {approx }}^{T}(l)=\sum_{n \in \mathbb{Z}} \frac{\tau}{T}(a[n]-a[n-1]) \beta^{1}\left(\frac{r t}{T}-n+\frac{1}{2}\right) .
$$

For the first example, $T=0.2$, and using $H_{1}, G_{3 / 4}, G_{2 / 3}$, and $H_{1 / 2}$, this formula gives $L^{2}[-3,3]$-norm error equal to $1.1 \times$ $10^{-2}, 2.1 \times 10^{-2}, 2.7 \times 10^{-2}$, and $5.1 \times 10^{-2}$, respectively.

\section{CONCLUSION}

In this work, we have studied the approximation of functions from their samples by using quasi-interpolation formulas. These schemes give a good quality since they have a certain order of approximation, and they are numerically efficient sinee the necessary prefiltering of the samples can be done with little support. In particular, they give a quality close to that given by the classical interpolating formula.

A rational filter bank with a rate $r<1$ can be used for the prefiltering of the samples, maintaining the approximation order and the little support. When the rate $r$ decreases, we lose in quality of approximation, but we gain in numerical efliciency. Thus, in applications where the speed is more relevant than the quality, it is advisable to use a rate $r<1$.

\section{APPENDIX}

In this Appendix, we include the proofs of the results in Sections II, III, and Section V.

Proof that (9) can be Represented by (12) and that (13) and (14) are Equivalent: First, we prove that for any $k \in \mathbb{Z}$, there exist unique numbers $n$ and $j$ satisfying

$$
k=n q+j p, \quad n \in \mathbb{Z}, \quad j \in\{0,1, \ldots, q-1\} .
$$

Indeed, since $q$ and $p$ are coprime, there exist $a, b \in \mathbb{Z}$ such that $1=a p+b q$. Then, $k=k a p+k b q$. Using that $k a$ can be writton as $k a=l q+j$, wilh $j \in\{0, \ldots, q-1\}$ and $l \in \mathbb{Z}$, we obtain (40) with $n=l p+k b$. The numbers $n$ and $q$ are unique since $n q+j p=\tilde{n} q+\tilde{j} p$ implies $(n-\tilde{n}) q=(\tilde{j}-j) p$, $\widetilde{j}-j \in\{-(q-1), \ldots, q-1\}$, and $q, p$ are coprime.

From the representation (40), we obtain that $H(z)=$ $\sum_{j=0}^{q-1} z^{-j p} G_{j}\left(z^{q}\right)=\sum_{j=0}^{q-1} \sum_{n \in \mathbb{Z}} g_{j}[n] z^{-(n q+j p)}$ if and only if $g_{j}[n]=h[n q+j p]$, for all $n \in \mathbb{Z}$ and $j \in\{0, \ldots, q-1\}$. Hence, (13) and(14) are equivalent.

Using that $g_{j}[n]=h[n q+j p]$, we obtain

$$
\begin{aligned}
& \sum_{j=0}^{q-1} \sum_{k \in \mathbb{Z}} f(q k T-j T) g_{j}[n-k p] \\
& \quad=\sum_{j=0}^{q-1} \sum_{k \in \mathbb{Z}} f([q k-j] T) h[q n-p(q k-j)] \\
& =\sum_{k \in \mathbb{Z}} f(k T) h(q n-p k) .
\end{aligned}
$$

Proof of Lemma 1: Notice first that $Q_{T}$ reproduecs the polynomials in $\Pi_{L-1}$ if and only if $Q_{r}$ does it since $Q_{T}=$ $\sigma_{T / r} Q_{r} \sigma_{r / T}$, where $\sigma_{a}$ denotes the operator $\sigma_{a} f=f(\cdot / a)$.

Notice that $\sum_{n \in \mathbb{Z}}(p n-j r)^{t} S_{j}(t-p n)$ converges in the sense of tempered distributions (see [21, Theorem 4.3-1]). Applying the Fourier transform, we obtain that

$$
t^{l}=\left[Q_{r} x^{t}\right](t)=\sum_{j=0}^{q-1} \sum_{n \in \mathbb{Z}}(p n-j r)^{t} S_{j}(t-p n)
$$

if and only if

$$
\frac{\delta^{(l)}(w)}{(-2 \pi i)^{l}}=\sum_{j=0}^{q-1} \sum_{n \in \mathbb{Z}}(p n-j r)^{l} e^{-2 \pi i p n w} \hat{S}_{j}(w)
$$


TABLE $]$

$L^{2}[-3,3]-$ NORM OF THE ERROR

\begin{tabular}{|c|c|c|c|c|c|c|c|c|}
\hline & $H^{\text {int }}$ & $H_{1}^{o}$ & $H_{1}$ & $G_{3 / 4}$ & $G_{2 / 3}$ & $H^{\mathrm{IIR}}$ & $H_{1 / 2}^{o}$ & $H_{1 / 2}$ \\
\hline$f(t)$ & $3.8 \times 10^{-4}$ & $4 \times 10^{-4}$ & $7 \times 10^{-4}$ & $2.6 \times 10^{-3}$ & $3.2 \times 10^{-3}$ & $3.6 \times 10^{-3}$ & $4 \times 10^{-3}$ & $6.4 \times 10^{-3}$ \\
$f(t) \cos (3 t)$ & $3.2 \times 10^{-3}$ & $3.1 \times 10^{-3}$ & $7.2 \times 10^{-3}$ & $2.3 \times 10^{-2}$ & $3.2 \times 10^{-2}$ & $4.1 \times 10^{-2}$ & $4.4 \times 10^{-2}$ & $6.1 \times 10^{-2}$ \\
$f(t)+u(t-1)$ & 0.26 & 0.26 & 0.25 & 0.2 & 0.25 & 0.21 & 0.47 & 0.26 \\
\hline
\end{tabular}
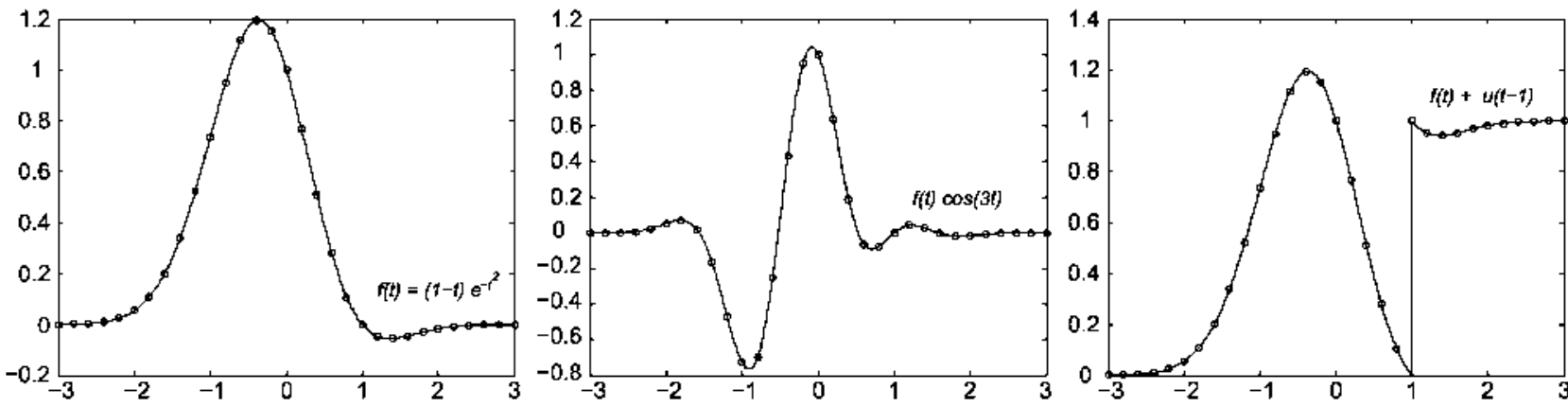

Fig. 6. Examples.
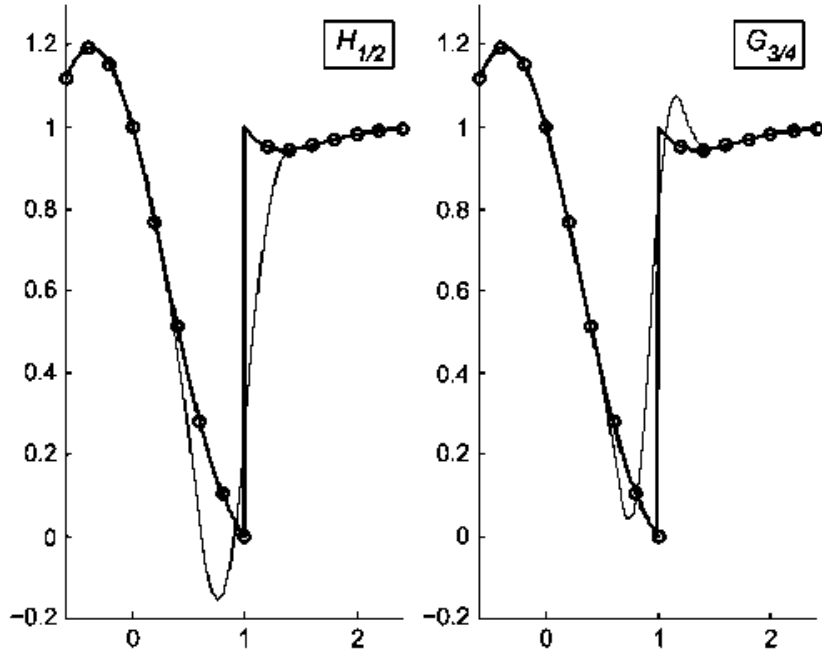

Fig. 7. Function $f(t)+u(t-1)$ and the reconstrueted functions from samples taken with period $T=0.2$ using $H_{1 / 2}$ and $G_{3 / 4}$, respectively.
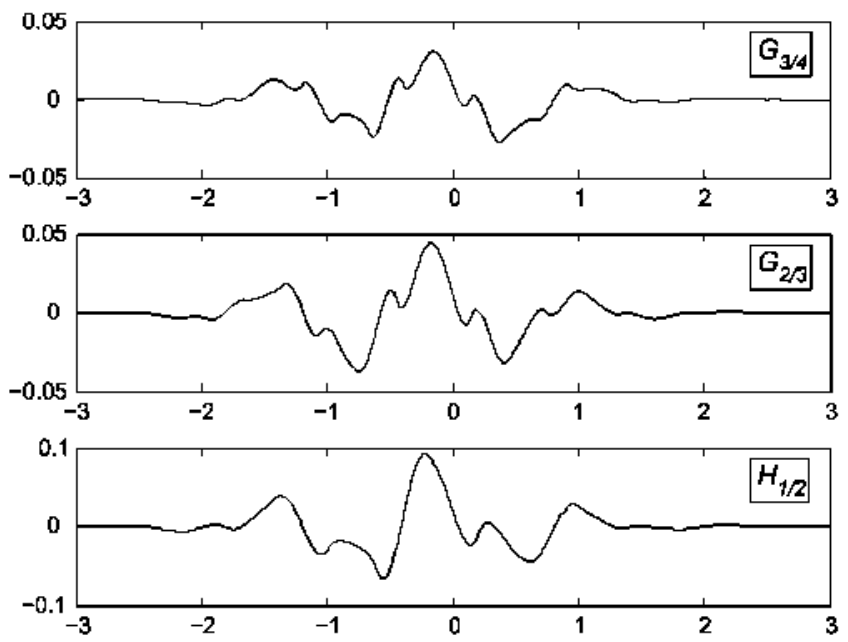

Fig. 8. Pointwise error in the reconstruction of the second example $f(t) \cos (3 t)$ using $G_{3 / 1}, G_{2 / 3}$ and $H_{1 / 3}$.
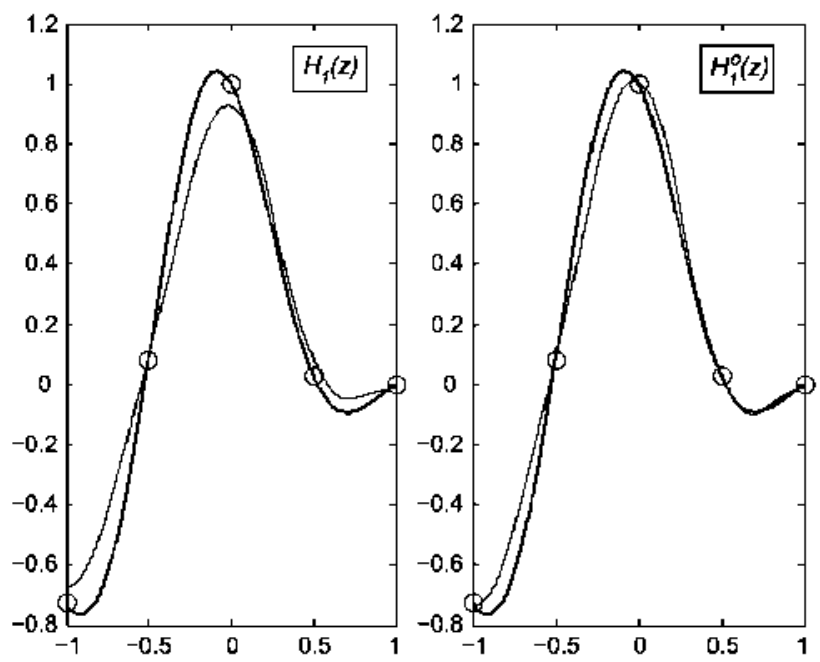

Fig. 9. Function $f(t) \cos (3 t)$ and the reconstructed functions from samples taken with period $t=0.5$ using the filters $H_{1}$ and $H_{1}^{\circ}$ respectively.

where $\delta$ denotes the Dirac delta. The Fourier transform of $(x-$ $j r)^{l}$ has compact support. Thus, applying the Poisson formula (see [22]), we obtain that the above equality holds if and only if

$$
p \delta^{(l)}(w)=\sum_{j=0}^{q-1} \sum_{n \in \mathbb{Z}} e^{-2 \pi i j(r w)-n / q)} \delta^{(l)}\left(w-\frac{n}{p}\right) \hat{S}_{j}(w)
$$

or equivalently if

$$
\begin{aligned}
\delta_{n} p \delta^{(l)}(w)= & \sum_{j=0}^{q-1} e^{-2 \pi i j(r w-n / q)} \delta^{(l)}\left(w-\frac{n}{p}\right) \hat{S}_{j}(w) \\
= & \sum_{j=0}^{q-1} e^{-2 \pi i j(r w-n / q)} \delta^{(l)}\left(w-\frac{n}{p}\right) \\
& \times G_{j}\left(e^{2 \pi i w}\right) \hat{\varphi}(w)
\end{aligned}
$$

for all $n \in \mathbb{Z}$. Taking into account that for any function $\xi \in$ $C^{\infty}(\mathbb{R})$ we have $\xi(w) \delta^{(l)}(w-n / p)=0$, for $l=0,1, \ldots, L-1$, 
if and only if $\xi(w+n / p)=O\left(w^{L}\right)$, we dednce that the above equality holds for every $l=0,1, \ldots, L-1$, or equivalently $Q_{r}$ reproduces polynomials of $\Pi_{L-1}$, if and only if condition (16) is satisfied for all $n \in \mathbb{Z}$.

Proof of Lemma 2: Let $z$ denote $z=e^{2 \pi i w}$. A sufficient condition (also necessary in most of the times) for (16) is

$$
\begin{aligned}
\mathbf{e}_{q}^{\top}\left(z^{r}\right) \mathbf{G}\left(z e^{2 \pi i n / p}\right) & =O\left(w^{L}\right), \quad n=1, \ldots, p-1 \\
\mathbf{e}_{q}^{\top}\left(z^{r}\right) \mathbf{G}(z) \hat{\varphi}(w)-p & =O\left(w^{L}\right) .
\end{aligned}
$$

Since we have assumed that $\varphi$ satisfies the Strang-Fix conditions of order $L$ and has compact support, we have

$$
1-\overline{\hat{\varphi}}_{d}(w) \hat{\varphi}(w)=1-\frac{|\hat{\varphi}(w)|^{2}}{\sum_{n \in \mathbb{Z}}|\hat{\varphi}(w+n)|^{2}}=O\left(w^{2 L}\right)
$$

(see [8, inequality (53)]). Hence, condition (41) is equivalent to(17).

In terms of the polyphase components (15), the condition( 17 ) reads as, for $n=0,1, \ldots, p-1$

$$
\sum_{k=0}^{p-1} C_{k}(w) a^{n(p-1-k)}=p \overline{\hat{\varphi}}_{d}(w) \delta_{\vartheta}+O\left(w^{L}\right)
$$

where $C_{k}(w)=\sum_{j=0}^{q-1} R_{k, j}\left(z^{p}\right) z^{-j r-(p-1-k)}, z=e^{2 \pi i w}$ and $a=e^{-2 \pi i / p}$, or equivalently, for $n=0,1, \ldots, p-1$

$$
\sum_{k=0}^{p-1} C_{k}^{(l)}(0) a^{n(p-1-k)}=p \overline{\hat{\varphi}}_{d}^{(l)}(0) \delta_{n}, \quad l=0, \ldots, L-1
$$

Denoting $\mathbb{C}=\left[C_{k}^{(l)}(0)\right]_{l=0, \ldots, L-1, k=0, \ldots, p-1}$ and $\mathrm{A}=$ $\left[a^{n(p-1-k)}\right]_{k=0, \ldots, p-1, n=0, \ldots, p-1}$, the above equalities read as

$$
\mathrm{CA}=p\left[\hat{\varphi}_{d}^{(l)}(0) \delta_{n}\right]_{l=0, \ldots, L-1, n=0, \ldots, p-1} .
$$

Using that $A^{*}=p 0$, where $A^{*}$ denotes the transpose conjugate of $A$ and $\mathbf{I}$ the identity matrix, we deduce that the above equality holds if and only if $C_{k}^{(l)}(0)=\overline{\hat{\varphi}}_{d}^{(l)}(0)$ for $k=0, \ldots, p-1, l=0, \ldots, L-1$, which proves $(18)$.

Proof of Theorem 1: Let $P_{t}^{k}(x)$ denotes the Taylor polynomial of degree $k$ for $f$ at $t$. Any function $f$ in the Sobolev space $W_{\infty}^{L+1}(\mathbf{R})$ satisfies (see [20, Ch. 2])

$$
\begin{aligned}
f(x) & =P_{t}^{L}(x)+R_{t}^{L}(x) \\
& =\frac{f^{(L)}(t)}{L !}(x-t)^{L}+P_{t}^{L-1}(x)+R_{t}^{L}(x)
\end{aligned}
$$

where the remainder $R_{t}^{L}$ can be expressed as

$$
R_{t}^{L}(x)=\frac{(x-t)^{L+1}}{L !} \int_{0}^{1}(1-\tau)^{L} f^{(L+1)}(t+\tau[x-t]) d \tau
$$

Since $Q_{T}$ reproduces polynomials in $\Pi_{L-1}$, we have $f(t)=P_{t}^{L-1}(t)=Q_{T} P_{t}^{L-1}(t)$. Hence, using the notation $\left[Q_{T} \xi(x)\right](t)$ for $Q_{T} \xi(t)$, we obtain

$$
\begin{aligned}
Q_{T} f(t)-f(t) & =Q_{T}\left(f-P_{t}^{L-1}\right)(t) \\
& =\left[Q_{T}\left\{\frac{f^{(L)}(t)}{L !}(x-t)^{L}+R_{t}^{L}(x)\right\}\right](t) \\
& =\frac{f^{(L)}(t)}{L !}\left[Q_{T}(x-t)^{L}\right](t)+Q_{T} R_{t}^{L}(t) .
\end{aligned}
$$

We have

$$
\begin{aligned}
{\left[Q_{T}(x-t)^{L}\right](t) } & =\sum_{j=0}^{q-1} \sum_{n \in \mathbb{Z}}(q n T-j T-t)^{L} S_{j}\left(\frac{r t}{T}-n p\right) \\
& =\frac{T^{L}}{r^{L}} \Omega\left(\frac{r t}{T}\right) .
\end{aligned}
$$

Besides, taking into account that

$$
\left|R_{t}^{L}(q n T-j T)\right| \leq \frac{T^{L+1}}{r^{L+1}} \frac{\left\|f^{(L+1)}\right\|_{\infty}}{(L+1) !}\left(\frac{r}{T} t-p n+j r\right)^{L+1}
$$

and that $\sum_{j=0}^{q-1} \sum_{n \in \mathbb{Z}}|t-p n+j v|^{L+1}\left|S_{j}(t-p n)\right|$ is a bounded function, we obtain

$$
\begin{aligned}
Q_{T} R_{t}^{L}(t) & =\sum_{j=0}^{q-1} \sum_{n \in \mathbb{Z}} R_{t}^{L}(q n T-j T) S_{j}\left(\frac{r t}{T}-p n\right) \\
& =O\left(T^{L+1}\right) .
\end{aligned}
$$

From this estimation, (42), and (43), the estimation (29) follows.

Similarly, we can prove that for $f \in W_{\infty}^{L}(\mathbf{R})$, the remainder $R_{t}^{L-1}(x)=f(x)-P_{t}^{L-1}(x)$ satisfies

$$
\left|Q_{T} R_{t}^{L-1}(t)\right| \leq \frac{\|\widetilde{\Omega}\|_{\infty}}{L !}\left\|f^{(L)}\right\|_{\infty} \frac{T^{L}}{r^{L}}
$$

which, as $Q_{T} f(t)-f(t)=Q_{T}\left(f-P_{t}^{L-1}\right)(t)=Q_{T} R_{t}^{L-1}(t)$, proves (28).

Proof that (31) Holds With E(W) Given by (32): It can be easily verified that the approximation operator $Q_{T}$ can be expressed by

$$
Q_{T} f(t)=\sum_{n \in \mathbb{Z}} \int f(\tau) \tilde{\Phi}^{\top}\left(\frac{\tau}{h}-p n\right) \Phi\left(\frac{t}{h}-n p\right) \frac{d \tau}{h}
$$

where $h=T / r, \Phi=\left[\phi_{0}, \ldots, \phi_{p-1}\right]^{\top}, \widetilde{\Phi}=\left[\widetilde{\phi}_{0}, \ldots, \widetilde{\phi}_{p-1}\right]^{\top}$, and

$$
\begin{aligned}
\phi_{s}(t) & =\varphi(t-s), \\
\widetilde{\phi}_{s}(t) & =\sum_{j=0}^{q-1} \sum_{k \in \mathbb{Z}} g_{j}[k p+s] \delta(t+p k+j r)
\end{aligned}
$$


for $s=0,1, \ldots, p-1$. By applying $[10$, Theorem 1], it follows that $\mathcal{L}_{h}:=Q_{h r}$ satisfies (30), where the kernel is given by

$$
\begin{aligned}
E(w) & =\frac{1}{p^{2}}|p-\overline{\tilde{\tilde{\Phi}}}(w) \hat{\Phi}(w)|^{2}+\frac{1}{p^{2}} \sum_{n \neq 0}\left|\overline{\tilde{\tilde{\Phi}}}(w) \hat{\Phi}\left(w+\frac{n}{p}\right)\right|^{2} \\
& =1-\frac{2}{p} \Re[\overline{\hat{\tilde{\Phi}}}(w) \hat{\Phi}(w)]+\frac{1}{p^{2}} \sum_{n \in \mathbb{Z}}\left|\overline{\hat{\tilde{\Phi}}}(w) \hat{\Phi}\left(w+\frac{n}{p}\right)\right|^{2} .
\end{aligned}
$$

Denoting $z=e^{2 \pi i w}$, for $n \in \mathbb{Z}$, we have

$$
\begin{aligned}
\overline{\hat{\Phi}}(w) \hat{\Phi}\left(w+\frac{n}{p}\right)= & \sum_{s=0}^{p-1} \sum_{j=0}^{q-1} z^{-j r} \sum_{k \in \mathbb{Z}} g_{j}[k p+s] z^{-p k} \\
& \times e^{-2 \pi i s(w+n / p)} \hat{\varphi}\left(w+\frac{n}{p}\right) \\
= & \sum_{j=0}^{q-1} z^{-j r} \sum_{s=0}^{p-1} \sum_{k \in \mathbb{Z}} g_{j}[k p+s] \\
& \times\left(z e^{2 \pi i n / p}\right)^{-p k-s} \hat{\varphi}\left(w+\frac{n}{p}\right) \\
= & \sum_{j=0}^{q-1} z^{-j r} G_{j}\left(z e^{2 \pi i n / p}\right) \hat{\varphi}\left(w+\frac{n}{p}\right) \\
= & \mathbf{e}_{q}^{\top}\left(z^{r}\right) \mathbf{G}\left(z e^{2 \pi i n / p}\right) \hat{\varphi}\left(w+\frac{n}{p}\right) .
\end{aligned}
$$

Then

$$
\begin{aligned}
\sum_{n \in \mathbb{Z}} & \left|\overline{\tilde{\Phi}}(w) \hat{\Phi}\left(w+\frac{n}{p}\right)\right|^{2} \\
& =\sum_{n \in \mathbb{Z}}\left|\mathbf{e}_{q}^{\top}\left(z^{r}\right) \mathbf{G}\left(z e^{2 \pi i n / p}\right) \hat{\varphi}\left(w+\frac{n}{p}\right)\right|^{2} \\
& =\sum_{s=0}^{p-1} \sum_{n \in \mathbb{Z}}\left|\mathbf{e}_{q}^{\top}\left(z^{r}\right) \mathbf{G}\left(z e^{2 \pi i s / p}\right) \hat{\varphi}\left(w+n+\frac{s}{p}\right)\right|^{2} \\
& =\sum_{s=0}^{p-1}\left|\mathbf{e}_{q}\left(z^{r}\right) \mathbf{G}\left(z e^{2 \pi i s / p}\right)\right|^{2} A_{\varphi}\left(w+\frac{s}{p}\right) .
\end{aligned}
$$

Using this equality and (45), the expression for the integral kernel (32) follows. From (30), with $h=T / r$, (31) follows. Notice that by applying [10, Lemma 2] using the expression(46), we obtain a new proof of the lemma

Proof of Theorem 2: We use the notation of the above proof. As $\sum_{n \in \mathbb{Z}} \sum_{s=0}^{p-1}|x-s-n p|^{2 L} \phi_{s}(x-n p)$ is bounded (since $\varphi$ is continuous and has compact support), and $\int\left|\widetilde{\phi}_{s}(t)\right| d t$ and $\int|t-n|^{2 L}\left|\widetilde{\phi}_{s}(t)\right| d t$ are bounded (since $g_{j}$ are FIR filters), the hypothesis of [10, Theorem 3] holds for $\mathcal{L}_{h}:=Q_{h r}$. By applying this theorem and Lemma 1, the first assertion of Theorem 2 follows. As the integral kernel $E(w)$ has, in this case, infinite bounded derivatives, the estimation (33) follows from [10, Theorem 4], taking into account (46). Finally, the inequality (34) follows from [10, Theorem 5].

\section{ACKNOWLEDGMENT}

The author wishes to thank the referees for their valuable and constructive comments and A. G. García for his kind reading of the manuscript and for the suggested changes.

\section{REFERENCES}

[1] P. Thévenaz, T. Blu, and M. Unser, "Interpolation revisited," IEEE Trans, Med. Imag., vol. 19, no. 7, pp. 739-758, Jul. 2000.

[2] M. Unser, "Sampling-50 years after Shannon," Proc. IEEE, vol. 88, no. 4 , pp. $569-587$, Apr. 2000.

[3] A. Aldroubi and M. Unser, "Sampling procedures in funtion spaces and asymptotic equivalence with Shannon's sampling theory," Numer. Funct. Anal. Optimiz, vol. 15, no. 1, pp. 1-21, Feb. 1994.

[4] P. Thévenaz, T. Blu, and M. Unser, "Image interpolation and resampling," in Handbook of Medical Imaginig Processing and Analysis, I. N. Bankman, Ed. New York: Academic, 2000, pp. 393-420.

[5] T. Blu, P. Thévenaz, and M. Unser, "MOMS: Maximal-order interpolation of minimal support," IEEE Trans. Signal Process., vol. 10, no. 7. pp. 1069-1080, Jul. 2001.

[6] M. Unser, A. Aldroubi, and M. Eden, "B-spline signal processing: Part II-Efficient design and applications," IEEE Trans. Signal Process., vol. 41 , no. 2 , pp. 834-848, Feb. 1993.

[7] T. Blu and M. Unser, "Quantitative Fourier analysis of approximation techniques: Part I-interpolators and projectors, and Part II-wavelets," IEEE Trans. Signal Process, vol. 47, no. 10, pp. 2783-2806, Oct. 1999.

[8] M. Unser and I. Daubechies, "On the approximation power of convolution-based least squares versus interpolation," IEEE Trans. Signal Process., vol. 45, no. 7, pp. 1697-1711, Jul. 1997.

[9] J. J. Lei, R. Q. Jia, and E. W. Cheney, "Approximation from shiftinvariant spaces by integral operators," SIAM J. Math. Anal., vol. 28, no. 2, pp. 481-498, Mar. 1997.

[10] T. Blu and M. Unser, "Approximation error for quasi-interpolators and (multi-)wavelet expansions," Appl. Comput. Harmon. Anal., vol. 6, no. 2, pp. 219-251, Mar. 1999.

[11] M. Unser, "Quasi-orthogonality and quasi-projections," Appl. Comput. Harmon. Anal., vol. 3, no. 3, pp. 201-251, Jul. 1996.

[12] L. Condat, T. Blu, and M. Unser, "Beyond interpolation: Optimal reconstruction by quasi-interpolation," in Proc. IEEE Int. Conf. Image Process., Sep. 2005, vol. 1, pp. 33-36.

[13] L. Condat and A. Montanvert, "Analyse multirésolution $L^{2}$-optimale: Estimation par quasi-projections," in Proc. GRETSI, Sep. 2005, pp. $783-786$.

[14] L. Condat and D. Van De Ville, "Quasi-interpolating spline models for hexagonally-sampled data," IEEE Trans. Signal Process., vol. 16, no. 5, pp. 1195-1206, May 2007.

[15] A. G. García, M. A. Hérnandez-Medina, and G. Pérez-Villalón, "Oversampling in shift-invariant spaces with a rational sampling period," IEEE Trans. Signal Process., vol. 57, no. 9, pp. 3442-3449, Sep. 2009.

[16] L. Condat and A. Montanvert, "Reconstruction from non-uniform samples: A direct variatonal approach in shift-invariant spaces," Tech. Rep. hal-00338881, 2005.

[17] P. P. Vaidyanathan, Multirate Systems and Filter Banks. Englewood Cliffs, NJ: Prentice-Hall, 1993.

[18] M. Vetterli and J. Kovaĉević, Wavelets and Subband Coding. Englewood Cliffs, NJ: Prentice-Hall, 1995.

[19] C. K. Chui, An Introduction to Wavelets. New York: Academic, 1992.

[20] R. DeVore and G. G. Lorentz, Constructive Approximation. New York: Springer, 1993.

[21] A. H. Zemanian, Distribution Theory and Transform Analysis. New York: Dover, 1965

[22] C. Gasquet and P. Witomski, Fourier Analysis and Applications. New York: Springer, 1999.

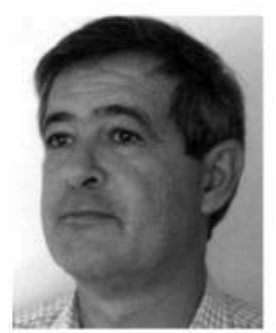

G. Pérez-Villalón was born in Teruel, Spain, in 1960. He received the M.Sc. degree in mathematics from the University Complutense of Madrid, Spain, in 1983 and the $\mathrm{Ph} . \mathrm{D}$. degree in mathematics from the University Carlos III de Madrid, Spain, in 2005.

$\mathrm{He}$ is currently an Associate Professor with the Department of Mathematics of the E.U.I.T.T., Madrid Polytechnic University, Madrid, Spain. His research interests include wavelets, interpolation, approximation, and sampling theory. 